A great deal of inspiration and guidance in this field has come from the other sciences, most notably biology, physics, and mathematics. Physicists were dealing with bistable systems and periodic oscillators long before it occurred to chemists that their reactions might behave in similar fashion. Periodic oscillation is ubiquitous in living systems and the hope that understanding simple chemical oscillators will provide insight into biological oscillators motivates many in this area. Similarly, spatial pattern formation in chemical systems may be of relevance in understanding cellular differentiation, chemotaxis, and related processes in biology. Certainly the similarities in appearance between the chemical waves of the type shown in Figure 18 and the aggregation pattern of the slime mold Dictyostelium discoidium ${ }^{73}$ are striking. In fact, Turing's seminal model ${ }^{74}$ for reaction-diffusion systems was inspired by the problem of biological morphogenesis.

Nearly all of the mathematical apparatus for dealing with new dynamical phenomena in chemistry has been available in the mathematical and physical literature before the phenomena were found experimentally. It was a mathematician, David Ruelle, ${ }^{75}$

(73) Tomchik, K. J.; Devreotes, P. N. Science 1981, 212, 443-6.

(74) Turing, A. M. Phil. Trans. R. Soc. (London), Ser. B 1952, 237, $37-72$.

(75) Ruelle, D. Trans. N.Y. Acad. Sci. 1973, 35, 66-71.

(76) Bazsa, G.; Epstein, I. R., unpublished. who predicted that chemical reactions could oscillate in a chaotic fashion several years before such behavior was experimentally established. As the chemical phenomena become better characterized, the mathematics provides a framework which highlights the fundamental identity of apparently dissimilar phenomena in chemistry, physics, biology, and even geology.

The safest way to predict the future is, of course, to extrapolate from the past. On this basis one should expect that new dynamical phenomena will be found in chemistry and that these phenomena will be describable with simple models and will occur in systems of apparently simple composition. One may further expect that these phenomena will have analogues in systems of interest to biologists and physicists and that the mathematical tools for their analysis already exist. Finally, one may certainly assume that complex dynamical phenomena will continue to provide many fascinating problems for physical chemists to ponder.

Acknowledgment. My own work in this area has been supported by the National Science Foundation through grants CHE7905911, CHE8204085, and INT8217658 and by a NATO Research Grant. I gratefully acknowledge the invaluable help of many able co-workers including Kenneth Kustin, Colin Steel, György Bazsa, Patrick De Kepper, Miklós Orbån, Jerzy Maselko, Mohamed Alamgir, Reuben Simoyi, Robert Olsen, Oscar Valdes-Aguilera, Debra Banville, and Christopher Dateo.

\title{
ARTICLES
}

\section{Intramolecular Vibrational Relaxation of Benzene}

\author{
E. Riedle, H. J. Neusser, ${ }^{*}$ E. W. Schlag, \\ Institut für Physikalische und Theoretische Chemie, Technische Universität München, D 8046 Garching, \\ West Germany
}

and S. H. Lin

Department of Chemistry, Arizona State University, Tempe, Arizona 85287 (Received: April 19, 1983)

\begin{abstract}
The purpose of this paper is to present a theory for the previously published high-resolution spectra of $14_{0}^{1} 1_{0}^{2}$ of $\mathrm{C}_{6} \mathrm{H}_{6}$. The analysis of these high-resolution spectra revealed an intramolecular process which was found to be strongly dependent on the rotation of the molecule. Here, possible intramolecular coupling processes are discussed and a qualitative comparison of theoretical results for the Coriolis coupling effect with the experiment is performed.
\end{abstract}

\section{Introduction}

Recently there has been considerable interest in the theoretical analysis of systems of coupled anharmonic oscillators. ${ }^{1-6}$ As the energy is increased, many classical systems are known to undergo a transition from regular to stochastic motion. Considerable effort in this area has been devoted to finding the phenomenology of the analogous quantum transition.

(1) D. W. Noid, M. L. Koszykowski, and R. A. Marcus, Annu. Rev. Phys. Chem., 31, 267 (1981).

(2) E. J. Heller and M. J. Davis, J. Phys. Chem., 86, 2118 (1982)

(3) I. Hamilton, D. Carter, and P. Brumer, J. Phys. Chem., 86, 2124 (1982)

(4) R. Kosloff and S. A. Rice, J. Chem. Phys., 74, 1340 (1981).

(5) R. M. Stratt, N. C. Handy, and W. H. Miller, J. Chem. Phys., 71, 3311 (1980).

(6) M. Shapiro and M. S. Child, J. Chem. Phys., 76, 6176 (1982).
In a previous paper, ${ }^{7}$ the density matrix method has been applied to treat coupled anharmonic system. For this purpose, the adiabatic approximation has been employed to find the basis set. ${ }^{8}$ It has been shown that the energy eigenvalues obtained from the adiabatic approximation are in very good agreement with exact ones $^{6,9}$ and are on the whole better than those obtained from the SCF calculation. ${ }^{9}$ Furthermore, the adiabatic approximation can provide analytical expressions of wave functions and energy eigenvalues of a coupled anharmonic system. Recently it has been shown by using the Schrödinger equation method that both de-

7) S. H. Lin, Chem. Phys. Lett., 86, 533 (1982)

(8) S. H. Lin, Chem. Phys. Lett., 70, 492 (1980); S. H. Lin, X. G. Zhang, Z. D. Qian, X. W. Li, and H. Eyring, Proc. Natl. Acad.Sci. U.S.A., 79, 1356 (1982).

(9) H. Kono, X. G. Zhang, Z. D. Qian, X. W. Li, and S. H. Lin, Mol Phys., 47, 713 (1983). 
phasing and amplitude of wave functions play important roles in determining the quantum stochasticity of coupled anharmonic oscillators. ${ }^{6}$ It should be noted that the density matrix method can serve this purpose very well; the diagonal elements of the density matrix of the system describe the evolution of the population of the system, while the off-diagonal elements of the density matrix provide information about the phase evolution of the system. When the density matrix method is used, the time-dependent behavior of the memory function will determine whether a system undergoes a regular motion or a stochastic motion.

A main purpose of this paper is to apply the theory of intramolecular vibrational relaxation (IVR) ${ }^{10,11}$ based on the application of the adiabatic approximation to the IVR of benzene.

In a recent work ${ }^{12}$ we have found experimental evidence for a rotationally selective intramolecular coupling mechanism. Sub-Doppler two-photon spectra with a high resolution of 200 $\mathrm{MHz}$ have been measured below and above the onset of "channel three". Briefly, the experiment reveals that above the onset of "channel three" the fluorescence from final states with $K \neq 0$ is efficiently quenched. This points to a Coriolis coupling of the excited vibration to another vibration by a rotation of the benzene molecule around the figure axis $\left(\hat{M}_{z}\right)$. Therefore Coriolis coupling is expected to be the dominant mechanism which leads to a communication of vibrational states within the molecule.

In this work the redistribution process will be considered theoretically. We shall apply the theory of intramolecular vibrational relaxation (IVR) ${ }^{8}$ to benzene where experimental information is available from our recent experiments.

\section{General Theory}

For convenience of discussion, we briefly outline the theory of IVR based on the use of the adiabatic approximation in this section. ${ }^{8}$

The Hamiltonian of a polyatomic molecule can in general be expressed as

$$
\hat{H}=\hat{T}_{Q}+\hat{T}_{r v}+h_{q}
$$

where

$$
\begin{gathered}
\hat{h}_{q}=\hat{T}_{q}+V(q, Q) \\
\hat{T}_{n}=\frac{\left(\hat{M}_{x}-\hat{m}_{x}\right)^{2}}{2 I_{x}}+\frac{\left(\hat{M}_{y}-\hat{m}_{y}\right)^{2}}{2 I_{y}}+\frac{\left(\hat{M}_{z}-\hat{m}_{z}\right)^{2}}{2 I_{z}}= \\
\sum_{u} \frac{\left(\hat{M}_{u}-\hat{m}_{u}\right)^{2}}{2 I_{u}}
\end{gathered}
$$

Here $q$ 's and $Q$ 's represent the vibrational coordinates of highfrequency modes and low-frequency modes, respectively and $\left(\hat{M}_{x}\right.$, $\left.\hat{M}_{y}, \hat{M}_{z}\right)$ and $\left(\hat{m}_{x}, \hat{m}_{y}, \hat{m}_{z}\right)$ denote the rotational and vibrational angular momenta, respectively. In eq $2.2, \hat{h}_{q}$ represents the Hamiltonian operator of $q$ 's.

According to the adiabatic approximation the solution of

$$
\hat{H} \psi_{v n l}=E_{v n l} \psi_{v n l}
$$

can be expressed as

$$
\begin{gathered}
\psi_{v n l}=\Psi_{v}(q, Q) \theta_{v n l}(Q, \chi) \\
\hat{h}_{q} \Psi_{v}(q, Q)=U_{v}(Q) \Psi_{v}(q, Q) \\
\left(\hat{T}_{Q}+T_{\mathrm{rv}}+U_{v}\right) \theta_{v n l}=E_{v n l} \theta_{v n l}
\end{gathered}
$$

where $n$ and $l$ denote the sets of the quantum numbers of lowfrequency modes and the quantum numbers of molecular rotation, respectively. Using the adiabatic approximation as a basis set, the IVR rate constant of single rovibrational level (SRVL) can be written as 8

$$
W_{v n l}=\frac{2 \pi}{\hbar} \sum_{v^{\prime} n^{\prime} l^{\prime}}\left|\left\langle\psi_{v^{\prime} n^{\prime} l^{\prime}}|\hat{H}| \psi_{v n l}\right\rangle\right|^{2} D\left(E_{v^{\prime} n^{\prime} l^{\prime}}-E_{v n l}\right)
$$

(10) R. P. Rettschnick in "Radiationless Transitions", S. H. Lin, Ed; Academic Press, 1980, pp 185-218.

(11) C. S. Parmenter, J. Phys. Chem., 86, 1735 (1982)

(12) E. Riedle, H. J. Neusser, and E. W. Schlag, J. Phys. Chem., 86, 4847 (1982). where $D\left(E_{v^{\prime} n^{\prime} l}-E_{u n l}\right)$ represents the line shape function

$D\left(E_{v^{\prime} n^{\prime} l^{\prime}}-E_{v n l}\right)=\frac{1}{\pi} \frac{\Gamma_{v^{\prime} n^{\prime} l^{\prime}, v n l}}{\Gamma_{v^{\prime} n^{\prime} l^{\prime}, v l n^{2}}+\left(E_{v^{\prime} n^{\prime} l^{\prime}}-E_{v n l}\right)^{2}}$

Here $\Gamma_{v^{\prime} n^{\prime}, v n l}$ denotes the dephasing (or damping) constants. $\hat{H}^{\prime}$ in eq 2.8 which represents the perturbations that induce IVR and can be attributed to the Born-Oppenheimer (BO) coupling

$$
\hat{H}_{\text {BO }}^{\prime}=\frac{1}{2} \sum_{\alpha} \hat{p}_{\alpha}^{2}=-\frac{\hbar^{2}}{2} \sum_{\alpha} \frac{\partial^{2}}{\partial Q_{\alpha}^{2}}
$$

i.e., the kinetic energy operator at low-frequency modes of vibration and to the Coriolis coupling

$$
\hat{H}_{\text {Cor }}^{\prime}=-\sum_{u} \frac{\hat{M}_{u} \hat{m}_{u}}{I_{u}}
$$

For example, if $V(q, Q)$ takes the form

$$
\begin{gathered}
V(q, Q)=\frac{1}{2} \sum_{i} \omega_{i}^{2} q_{i}^{2}+\frac{1}{2} \sum_{\alpha} \omega_{\alpha}^{2} Q_{\alpha}^{2}+\frac{1}{3 !} \sum_{i} \sum_{j} \sum_{\alpha} V_{i j \alpha} q_{i} q_{j} q_{\alpha}+ \\
\frac{1}{3 !} \sum_{i} \sum_{\alpha} \sum_{\beta} V_{i \alpha \beta} q_{i} Q_{\alpha} Q_{\beta}+\frac{1}{4 !} \sum_{i} \sum_{j} \sum_{\alpha} \sum_{\beta} V_{i j \alpha \beta} q_{i} q_{j} Q_{\alpha} Q_{\beta}+\ldots
\end{gathered}
$$

that is, including cubic and quartic anharmonic potentials, then we obtain

$$
\begin{aligned}
& U_{\mathrm{v}}(Q)= \\
& \sum_{i}\left[\left(v_{i}+1 / 2\right)-\chi_{i}\left(v_{i}+1 / 2\right)^{2}\right] \hbar \omega_{i}+1 / 2 \sum_{\alpha} \omega_{\alpha}\left(\left\{v_{i}\right\}\right)^{2} Q_{\alpha}\left(\left\{v_{i}\right\}\right)^{2}+\ldots
\end{aligned}
$$

where

$$
\begin{gathered}
Q_{\alpha}\left(\left\{v_{i}\right\}\right)=Q_{\alpha}+\sum_{i} \frac{\hbar V_{i j \alpha}}{6 \omega_{i} \omega_{\alpha}{ }^{2}}\left(v_{\mathrm{i}}+1 / 2\right)+\ldots \\
\omega_{\alpha}\left(\left\{v_{i}\right\}\right)^{2}=\omega_{\alpha}^{2}+\sum_{i} \frac{\hbar V_{i i \alpha \alpha}}{12 \omega_{i}}\left(v_{i}+1 / 2\right)+ \\
\sum_{i} \sum_{j} \frac{\hbar V_{i j \alpha}^{2}}{36 \omega_{i} \omega_{j}\left(\omega_{i}^{2}-\omega_{j}^{2}\right)}\left[\omega_{j}\left(v_{i}+1 / 2\right)-\omega_{i}\left(v_{\mathrm{j}}+1 / 2\right)\right]+\ldots
\end{gathered}
$$

It has been shown that ${ }^{8}$

$$
\left\langle\psi_{v^{\prime} n^{\prime} l^{\prime}}\left|\hat{H}_{\mathrm{BO}}^{\prime}\right| \psi_{v n l}\right\rangle=\sum_{i} \sum_{\alpha} \sum_{\beta} \frac{1}{3 !} V_{i \alpha \beta}\left\langle\psi_{v^{\prime} n^{\prime}}\left|q_{i} Q_{\alpha} Q_{\beta}\right| \psi_{v n l}\right\rangle+\ldots
$$

From eq 2.14-2.16 we can see that different types of anharmonic potentials play different roles in IVR: while the type (1/ 3!) $V_{i \alpha \beta} q_{i} q_{\alpha} Q_{\beta}$ plays the promoting role of the IVR of $q_{i}$, the type $(1 / 3 !) V_{i i \alpha} q_{i}^{2} Q_{\alpha}$ which introduces a vibrational coordinate shift in a totally symmetric mode so that the $Q_{\alpha}$ mode can accept more than one vibrational quantum in IVR determines the effectiveness of $Q_{\alpha}$ in accepting the vibrational excitation energy during IVR. Notice that the types $(1 / 4 !) V_{i i \alpha \alpha} q_{i}^{2} Q_{\alpha}^{2}$ and $(1 / 3 !) V_{i j \alpha} q_{i} q_{j} Q_{\alpha}$ can introduce the frequency shift in the $Q_{\alpha}$ mode so that the $Q_{\alpha}$ mode can accept more than one vibrational quantum in IVR.

In this paper, we are concerned only with the calculation of the SRVL IVR rate constant; this is sufficient for our purpose of interpreting the experimental data of benzene. ${ }^{12}$ For other purposes a distribution function for the low-frequency modes and molecular rotation may be required; in these cases the desired IVR rate constant can be obtained by multiplying the above-mentioned distribution function by the corresponding SRVL IVR rate constants. It has been shown numerically that the adiabatic approximation holds even for the case in which the frequency separation between the high-frequency mode and low-frequency modes is comparable. 6,9

\section{IVR of Benzene}

In this section we shall apply the results presented in section 2 to IVR of benzene, in particular of $14^{1} 1^{2}$ from a rotational level $(J K)$. 
Notice that the IVR rate in this case should consist of that contributed from $\hat{H}_{\mathrm{BO}}^{\prime}, W_{v n J K}^{(\mathrm{BO})}$ and of that contributed from $\hat{H}_{\text {Cor }}^{\prime}$, $W_{v n J K}^{\text {(Cor) }}$. We first consider $W_{v n J K}^{(\mathrm{BO})}$

$$
\begin{array}{r}
W_{v n J K}^{(\mathrm{BO})}=\frac{2 \pi}{\hbar} \sum_{n^{\prime} J^{\prime} K^{\prime} l \alpha \beta}\left|\sum_{\alpha \beta} \frac{1}{3 !} V_{i \alpha \beta}\left\langle\psi_{v^{\prime} n^{\prime} J^{\prime} K^{\prime}}\left|q_{i} Q_{\alpha} Q_{\beta}\right| \psi_{v n J K}\right\rangle\right|^{b} \times \\
D\left(E_{v^{\prime} n^{\prime} J^{\prime} K^{\prime}}-E_{i n j K}\right)
\end{array}
$$

Using the relations

$$
\begin{aligned}
\theta_{v n J K} & =Y_{\mathrm{JK}}(\chi) \prod_{\gamma} X_{v n_{\gamma}}\left(Q_{\gamma}\right) \\
\theta_{v^{\prime} n^{\prime} J^{\prime} K^{\prime}} & =Y_{J^{\prime} \mathrm{K}^{\prime}}(\chi) \prod_{\gamma} X_{v^{\prime} n_{\gamma}^{\prime}}\left(Q_{\gamma}\right)
\end{aligned}
$$

we obtain

$$
\begin{aligned}
W_{v n J K}^{\mathrm{BO})}=\frac{2}{\hbar^{2}} \sum_{i \alpha \beta}\left|\frac{1}{3 !} V_{i \alpha \beta}\right|^{2}\left(\frac{\hbar V_{i}}{2 \omega_{i}}\right) \times \\
\\
\operatorname{Re} \int_{0}^{\infty} \mathrm{d} t e^{-i t \omega_{i}} K_{n_{\alpha}}(t) K_{n_{\beta}}(t) \prod_{\gamma}^{\gamma \neq \alpha, \beta} G_{n_{\gamma}}(t)
\end{aligned}
$$

where

$$
\begin{gathered}
K_{n_{\alpha}}(t)=\sum_{n_{\alpha}^{\prime}}\left|\left\langle X_{v^{\prime} n_{\alpha}}\left|Q_{\alpha}\right| X_{v n_{\alpha}}\right\rangle\right|^{2} \exp \left[i t\left(\omega_{n_{\alpha}^{\prime}}-\omega_{n_{\alpha}}\right)-t \Gamma_{v^{\prime} n_{\alpha^{\prime}}, v n_{\alpha}}\right] \\
G_{n_{\gamma}}(t)=\sum_{n_{\gamma}^{\prime}}\left|\left\langle X_{v^{\prime} n_{\gamma}{ }^{\prime}} \mid X_{v n_{\gamma}}\right\rangle\right|^{2} \exp \left[i t\left(\omega_{n_{\gamma^{\prime}}}-\omega_{n_{\gamma}}\right)-t \Gamma_{v^{\prime} n_{\gamma^{\prime}}, v n_{\gamma}}\right]
\end{gathered}
$$

As shown in section 2 , for nontotally symmetric modes, only frequency shifts can be introduced by anharmonic couplings: in this case, $G_{n_{\gamma}}(t)$ is given by (Appendix)

$$
\begin{aligned}
& G_{n_{\gamma}}(t)=G_{n_{\gamma}=0}(t) \exp \left[-n_{\gamma} t\left(-i \omega_{\gamma}+\Gamma_{\gamma}\right)\right] \times \\
& \sum_{m_{\gamma}=0}^{n_{\gamma}} \frac{n_{\gamma} !}{m_{\gamma} !\left[\left(\frac{n_{\gamma}-m_{\gamma}}{2}\right) !\right]^{2}}\left(\frac{b_{\gamma}}{2}\right)^{m_{\gamma}}\left(\frac{a_{\gamma}}{2}\right)^{m_{\gamma}-m_{\gamma}}
\end{aligned}
$$

where $G_{n_{\gamma}=0}(t)$ represents the function of $G_{n_{\gamma}}(t)$ at $n_{\gamma}=0$ and is defined by

$$
\begin{aligned}
& G_{n_{\gamma}=0}(t)=\frac{\exp \left[\frac{1}{2} i t\left(\omega_{\gamma}^{\prime}-\omega_{\gamma}\right)\right]}{\eta_{\gamma}\left[1+\rho_{\gamma} e^{-2 t\left(\Gamma_{\gamma}-i \omega_{\gamma}^{\prime}\right)}\right]^{1 / 2}} \\
& \omega_{\gamma}=\omega_{\gamma}\left(\left\{v_{i}\right\}\right) \quad \omega_{\gamma}^{\prime}=\omega_{\gamma}\left(\left\{v_{i}^{\prime}\right\}\right) \\
& \rho_{\gamma}=-\left(\frac{\omega_{\alpha}^{\prime}-\omega_{\alpha}}{\omega_{\alpha}{ }^{\prime}+\omega_{\alpha}}\right)^{2} \\
& n_{\gamma}=\frac{\omega_{\gamma}+\omega_{\gamma}^{\prime}}{\left(4 \omega_{\gamma} \omega_{\gamma}^{\prime}\right)^{1 / 2}} \\
& a_{\gamma}=\frac{\left(\omega_{\gamma}{ }^{\prime 2}-\omega_{\gamma}^{2}\right)}{\left(\omega_{\gamma}+\omega_{\gamma}\right)^{2}} \frac{\left[1-e^{-2 t\left(\Gamma_{\gamma}-i \omega_{\gamma}{ }^{\prime}\right)}\right]}{\left[1+\rho_{\gamma} e^{-2 t\left(\Gamma_{\gamma}-i \omega_{\gamma}{ }^{\prime}\right)}\right]} \\
& b_{\gamma}=\frac{2 e^{-t\left(\Gamma_{\gamma}-i \omega_{\gamma}\right)}}{\eta_{\gamma}{ }^{2}\left[1+\rho_{\gamma} e^{-2 t\left(\Gamma_{\gamma}-i \omega_{\gamma}^{\prime}\right)}\right]}
\end{aligned}
$$

If $Q_{\alpha}$ is nontotally symmetric, then $K_{n_{\alpha}}(t)$ can be expressed in terms of $G_{n_{\alpha}}(t)$;

For totally symmetric modes, both vibrational coordinates and frequencies can be shifted by anharmonic couplings. The vibrational coordinate shifts are usually more important in IVR than vibrational frequency shifts. If the effect of vibrational frequency shifts are ignored, $G_{n_{\beta}}(t)$ defined by eq 3.6 can be expressed as

$$
\begin{gathered}
G_{n_{\beta}}(t)=\exp \left\{-\frac{1}{2} \Delta_{\beta}{ }^{2}\left[1-e^{t\left(i \omega_{\beta}-\Gamma_{\beta}\right)}\right]\right\} \times \\
\sum_{m_{\beta}=0}^{n_{\beta}} \frac{n_{\beta} !}{m_{\beta} !\left[\left(n_{\beta}-m_{\beta}\right) !\right]^{2}}\left\{\frac{1}{2} \Delta_{\beta}^{2}[1-\right. \\
\left.\left.e^{t\left(i \omega_{\beta}-\Gamma_{\beta}\right)}\right]^{2}\right\}^{n_{\beta}-m_{\beta}} \exp \left[-i t \omega_{\beta}\left(n_{\beta}-m_{\beta}\right)-t\left(n_{\beta}+m_{\beta}\right) \Gamma_{\beta}\right]
\end{gathered}
$$

where

$$
\Delta_{\beta}^{2}=\frac{\omega_{\beta}}{\hbar}\left[\sum_{i} \frac{\hbar V_{i i \beta}}{6 \omega_{i} \omega_{\beta}^{2}}\left(v_{i}^{\prime}-v_{i}\right)\right]^{2}
$$

To apply the above results for $W_{v n J K}^{(\mathrm{BO})}$ to a SRVL IVR rate of $14^{1}$ $1^{2}$ we should notice that $n_{\beta}=2, n_{\alpha}=0, n_{\gamma}=0$, and $v_{i}=1$ (i.e., $v_{i}=1 \rightarrow v_{i}^{\prime}=0$ ). In this case, we have

$$
K_{n_{\alpha}=0}(t)=\frac{\hbar}{2 \omega_{\alpha}} e^{t\left(\Gamma_{\alpha}+i \omega_{\alpha}\right)} G_{n_{\alpha}=1}(t)
$$

Here $G_{n_{\alpha}=1}(t)$ can be obtained from eq 3.7. From eq 3.4 we can see that the type of anharmonic potentials $(1 / 3 !) V_{i \alpha \beta} q_{i} Q_{\alpha} Q_{\beta}$ plays the promoting role in $W_{v n J k}^{(\mathrm{BO})}$. Thus if $q_{i}$ refers to the $\nu_{14}$ mode $\left(\mathrm{B}_{2 \mathrm{u}}\right)$ then the symmetry condition requires that $\left(Q_{\alpha}, Q_{\beta}\right)$ can have only $\left(A_{2 g}, B_{1 u}\right),\left(E_{1 g}, E_{2 u}\right)$, and $\left(E_{1 u}, E_{2 g}\right)$ symmetry. Notice that for $\mathrm{C}_{6} \mathrm{H}_{6}$ we have $\nu_{3}\left(\mathrm{~A}_{2 \mathrm{~g}}\right)=1210 \mathrm{~cm}^{-1} ; \nu_{12}\left(\mathrm{~B}_{1 \mathrm{u}}\right)=995 \mathrm{~cm}^{-1} ; \nu_{10}\left(\mathrm{E}_{1 \mathrm{~g}}\right)$ $=585 \mathrm{~cm}^{-1} ; \nu_{16}\left(\mathrm{E}_{2 \mathrm{u}}\right)=237 \mathrm{~cm}^{-1} ; \nu_{17}\left(\mathrm{E}_{2 \mathrm{u}}\right)=712 \mathrm{~cm}^{-1} ; \nu_{18}\left(\mathrm{E}_{1 \mathrm{u}}\right)$ $=923 \mathrm{~cm}^{-1} ; \nu_{19}\left(\mathrm{E}_{1 \mathrm{u}}\right)=1470 \mathrm{~cm}^{-1} ; \nu_{6}\left(\mathrm{E}_{2 \mathrm{~g}}\right)=522 \mathrm{~cm}^{-1} ; \nu_{8}\left(\mathrm{E}_{2 \mathrm{~g}}=\right.$ $1469 \mathrm{~cm}^{-1} ; \nu_{9}\left(\mathrm{E}_{2 g}\right)=1148 \mathrm{~cm}^{-1}$. On the other hand, if $q_{i}$ refers to the $v_{1}$ mode $\left(A_{1 g}\right)$, then as long as $Q_{\alpha}$ and $Q_{\beta}$ belong to the same symmetry, the symmetry condition in $(1 / 3 !) V_{j \alpha \beta} q_{i} Q_{\alpha} Q_{\beta}$ is satisfied.

In obtaining $W_{v n K K}^{(\mathrm{BO})}$ given by eq 3.4 the rotational effect has been ignored. The rotational effect on IVR in $W_{v n J K}^{(\mathrm{BO})}$ can be caused by the rotation-vibration coupling due to the vibrational coordinate dependence of $\left(I_{x}, I_{y}, I_{z}\right)$ and by the effect of the Coriolis coupling on initial and final states involved in IVR. To this approximation, $W_{v n J K}^{(\mathrm{BO})}$ is independent of $J K$.

Next we consider $W_{v n J k}^{\operatorname{cor}}$. It should be noted that for benzene, $\hat{M}_{z}$ belongs to $\mathrm{A}_{2 g}$, while $\left(\hat{M}_{x}, \hat{M}_{y}\right)$ belong to $\mathrm{E}_{1 g}$. Thus we shall discuss the calculation of $W_{v n J K}^{(\operatorname{cor})}$ due to the $\hat{M}_{z}$ component of $\hat{H}_{\text {Cor }}^{\prime}$ and due to the $\left(\hat{M}_{x}, \hat{M}_{y}\right)$ components of $\hat{H}_{\text {Cor }}^{\prime}$ separately.

For the $\hat{M}_{z}$ component of $\hat{H}_{\text {Cor }}^{\prime}$, using eq 3.2 and 3.3 and the relations

$$
\begin{gathered}
\hat{H}_{\mathrm{Cor}}^{\prime}\left(\hat{M}_{z}\right)=-\left(\hat{M}_{z} \hat{m}_{z}\right) / I_{z} \\
\hat{m}_{z}=\sum_{i \alpha} \zeta_{i \alpha}^{(z)}\left(q_{i} \hat{p}_{\alpha}-\hat{p}_{i} Q_{\alpha}\right)
\end{gathered}
$$

we obtain

$$
\begin{gathered}
W_{v n J K}^{(\mathrm{Cor})}\left(\hat{M}_{z}\right)=-\frac{2}{\hbar^{2}} \sum_{i \alpha}\left(\zeta_{\alpha \alpha}^{z z} \frac{K \hbar}{I_{z}}\right)^{2}\left(\frac{V_{i} \hbar^{2}}{4}\right)\left(2+\frac{\omega_{\alpha}}{\omega_{i}}+\frac{\omega_{i}}{\omega_{\alpha}}\right) \times \\
\operatorname{Re} \int_{0}^{\infty} \mathrm{d} t \exp \left[t\left(-i \omega_{i}+i \omega_{\alpha}+\Gamma_{\alpha}\right)\right] G_{n_{\alpha}=1}(t) G_{n_{\beta}=2}(t) \times \\
\prod_{\gamma}^{\gamma \neq \alpha, \beta} G_{n_{\gamma}=0}(t)
\end{gathered}
$$

where $G_{n_{\alpha}=1}(t)$ and $G_{n_{\beta}=2}(t)$ should be calculated from eq 3.7 and 3.14 , respectively. From eq 3.19 we can see that for the case in which the IVR is induced by the $\hat{M}_{z}$ component of the Coriolis coupling, the SRVL IVR rate is proportional to $K^{2}$.

Now we consider the calculation of $W_{v n J K}^{(\text {cor })}$ due to the $\left(\hat{M}_{x}, \hat{M}_{y}\right)$ components of $\hat{H}_{\text {Cor. }}^{\prime}$. Noticing that in this case $I_{x}=I_{y}$, we obtain

$$
W_{v n d K}^{(\operatorname{Cor})}\left(\hat{M}_{x}, \hat{M}_{y}\right)=W_{v n J K}^{(\operatorname{Cor})}(+)+W_{v n J K}^{(\operatorname{Cor})(-)}
$$

where $W_{V n J K}^{(\mathrm{Cor})}(+)$ represents the IVR rate constant for $(J K) \rightarrow(J$ $K+1$ )

$$
\begin{aligned}
& W_{v n J K}^{\operatorname{Cor})}(+)=\frac{2}{\hbar^{2}}\left(\frac{\hbar}{2 I_{x}}\right)^{2}\left(\frac{V_{i} \hbar^{2}}{4}\right)(J-K)(J+K+ \\
& \text { 1) } \sum_{i \alpha}\left(\left|\zeta_{i \alpha}^{(x)}\right|^{2}+\left|\zeta_{i \alpha}^{(y)}\right|^{2}\right)\left(2+\frac{\omega_{i}}{\omega_{\alpha}}+\frac{\omega_{\alpha}}{\omega_{i}}\right) \operatorname{Re} \int_{0}^{\infty} \mathrm{d} t \exp \left[t \left(-i \omega_{i}+\right.\right. \\
& \left.\left.i \omega_{\alpha}+\Gamma_{\alpha}\right)+i t(C-B)(2 K+1)\right] G_{n_{\alpha}=1}(t) G_{n_{\beta}=2}(t) \prod_{\gamma}^{\gamma \neq \alpha, \beta} G_{n_{\gamma}^{\prime}=0}(t)
\end{aligned}
$$




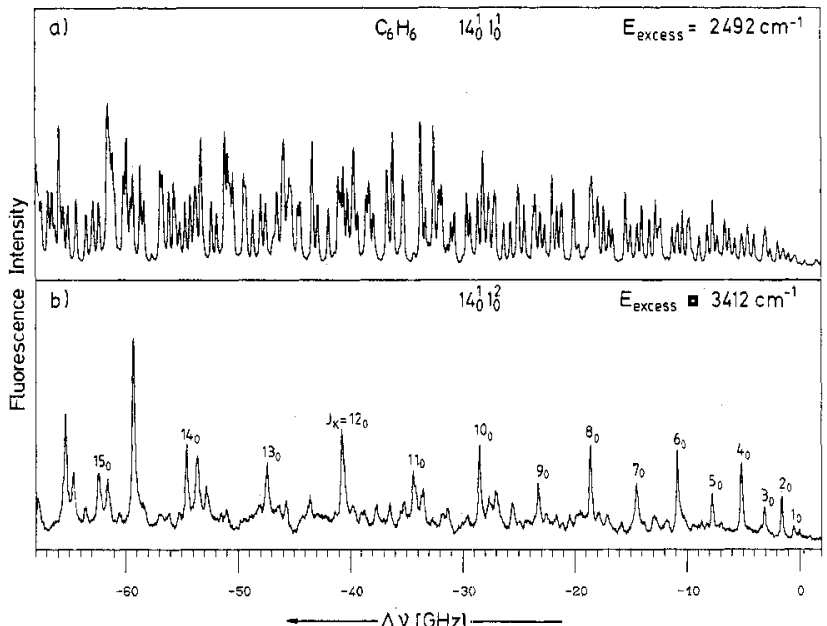

Figure 1. Doppler-free two-photon fluorescence excitation spectra of $\mathrm{C}_{6} \mathrm{H}_{6}:{ }^{12}$ (a) part of the $\mathrm{Q}$ branch of the $14_{0}^{1} \mathrm{l}_{0}^{1}$ band at an excess energy of $2492 \mathrm{~cm}^{-1} ;$ (b) corresponding part of the $14_{0}^{1} 1_{0}^{2}$ band at $3412 \mathrm{~cm}^{-1}$. Indicated are the $K=0$ lines as identified previously. ${ }^{12}$

and $W_{v n J K}^{(\mathrm{Cor})}(-)$ represents the IVR rate constant for $(J K) \rightarrow(J K$ $-1)$

$$
\begin{aligned}
& W W_{V h K}^{\operatorname{Cor}}(-)=\frac{2}{\hbar^{2}}\left(\frac{\hbar}{2 I_{x}}\right)^{2}\left(\frac{V_{i} \hbar^{2}}{4}\right)(J+K)(J-K+ \\
& \text { 1) } \sum_{i \alpha}\left(\left|\zeta_{i \alpha}^{(x)}\right|^{2}+\left|\zeta_{i \alpha}^{(\nu)}\right|^{2}\right)\left(2+\frac{\omega_{i}}{\omega_{\alpha}}+\right. \\
& \left.\frac{\omega_{\alpha}}{\omega_{i}}\right) \operatorname{Re} \int_{0}^{\infty} \mathrm{d} t \exp \left[t \left(-i \omega_{i}+\right.\right. \\
& \left.\left.i \omega_{\alpha}+\Gamma_{\alpha}\right)+i t(C-B)(-2 K+1)\right] G_{n_{\alpha}=1}(t) G_{n_{\beta}=2}(t) \prod_{\gamma}^{\gamma \neq \alpha, \beta} G_{n_{\gamma}=0}(t)
\end{aligned}
$$

Here the following energy expression for a rigid rotator has been used

$$
E_{J K}=\hbar B J(J+1)+(C-B) \hbar K^{2}
$$

From the viewpoint of symmetry for the IVR of $14^{1} 1^{2}$ if $q_{i}$ refers to the $\nu_{14}$ mode which has a symmetry of $\mathrm{B}_{2 \mathrm{u}}$, then in the $\hat{M}_{z}$ component of $\hat{H}_{\text {Cor }}^{\prime}$ the $Q_{\alpha}$ modes are of the $\mathrm{B}_{1 \mathrm{u}}$ symmetry; in this case there is only $\nu_{12}=936 \mathrm{~cm}^{-1}$ which has frequency lower than $\nu_{14}=1567 \mathrm{~cm}^{-1}$. Similarly in the $\left(\hat{M}_{x}, \hat{M}_{y}\right)$ components of the $H_{\text {Cor }}^{\prime}$ the $Q_{\alpha}$ modes are of the $\mathrm{E}_{2 \mathrm{u}}$ symmetry; in this case $\nu_{16}=$ $237 \mathrm{~cm}^{-1}$ and $\nu_{17}=712 \mathrm{~cm}^{-1}$ can be the $Q_{\alpha}$ modes in $W_{v n J K}^{(\text {Cor) }}$. Next if $q_{l}$ refers to the $\nu_{1}$ mode which has a symmetry of $\mathrm{A}_{1 \mathrm{~g}}$, then in the $\hat{M}_{z}$ component of $\hat{H}_{\text {Cor }}^{\prime}$ the $Q_{\alpha}$ modes are of the $\mathrm{A}_{2 \mathrm{~g}}$ symmetry; in this case there is only one $\mathrm{A}_{2 \mathrm{~g}}$ mode, i.e., $\nu_{3}=1246 \mathrm{~cm}^{-1}$ which will not be very effective in promoting the IVR. Similarly in the $\left(\hat{M}_{x}, \hat{M}_{y}\right)$ components of $\hat{H}_{\text {Cor }}^{\prime}$, the $Q_{\alpha}$ modes are of the $\mathrm{E}_{1 \mathrm{~g}}$ symmetry; in this case the $\nu_{10}=585 \mathrm{~cm}^{-1}$ mode can be a promoting mode in $W_{v n J K}^{\text {(Cor) }}$.

From section 2, we can see that only totally symmetric modes can have vibrational coordinate and frequency displacements induced by anharmonic couplings; because of these displacements, the totally symmetric modes can accept in principle any number of quanta of excitation energy within the limitation of energy conservation. On the other hand, the vibrational modes of other symmetries can have only vibrational frequency displacements induced by anharmonic couplings and thus these modes can accept only even number of quanta of vibrational excitation energy, if that particular mode is initially not excited.

\section{Discussion}

In a recent work we presented high-resolution spectra of $\mathrm{C}_{6} \mathrm{H}_{6}$ in the "channel three" region of $\mathrm{C}_{6} \mathrm{H}_{6}{ }^{12}$ By means of a two-photon absorption from two pulsed counterpropagating light beams ${ }^{14}$ we obtained Doppler-free two-photon fluorescence excitation spectra

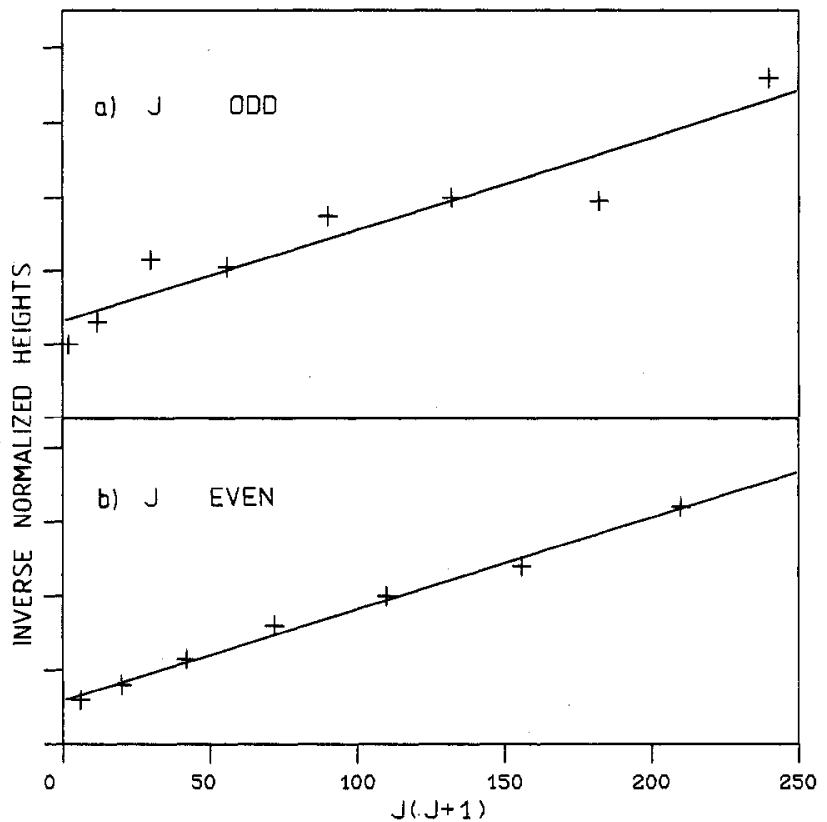

Figure 2. Inverse normalized heights of $K=0$ peaks plotted as a function of $J(J+1)$ as found from the measured spectrum in Figure 1b: (a) for $J$ odd; (b) for $J$ even. For discussion see text (eq 4.1). The solid lines are least-squares fits to the data points.

for several vibronic bands at different excess energies. The main results of this work are shown in Figure 1, a and b, for comparison. From this result it is clear that many spectral lines which are present in the $14_{0}^{1} 1_{0}^{1}$ spectrum are missing in the $14_{0}^{1} 1_{0}^{2}$ spectrum. In our recent work we concluded that the lack of spectral lines is due to the opening of a nonradiative channel by Coriolis coupling as the primary process which produces IVR.

Some spectral lines in the $14_{0}^{1} 1_{0}^{2}$ spectrum due to rovibronic transitions are assigned. A main feature here is that in the first part of the spectrum the $K=0$ lines are very pronounced. From section 3 we can see that the SRVL IVR rate due to the $\hat{M}_{z}$ component of $\hat{H}_{\text {Cor }}^{\prime}$ is proportional to $K^{2}$. Thus for the $K=0$ lines, the IVR rate due to this particular Coriolis coupling mechanism is zero and hence the fluorescence quantum yields for the $K=$ 0 lines are higher in this range since they do not undergo the subsequent nonradiative process. For the high-resolution spectra shown in Figure 1, each spectral line is due to a rovibronic transition and the intensity of each such spectral line in this case is proportional to the rotational Boltzmann factor and the statistical weights of the ground electronic state as well as to the fluorescence quantum yield of the particular single rovibronic level.

The nonradiative processes responsible for the quantum yield of fluorescence can be due to electronic relaxation and IVR. The SRVL IVR has been treated in section 3. From eq 3.4, 3.19, and 3.20 , we can see that the SRVL IVR rate for $K=0$ can be expressed as

$$
W_{v n J K=0}=W_{v n}^{(\mathrm{BO})}+J(J+1) W_{v n}^{(\mathrm{Cor})}
$$

where $W_{v n}^{(\mathrm{BO})}$ is given by eq 3.4 and $W_{u n}{ }^{(\mathrm{Cor})}$ is defined by

$$
\begin{aligned}
& W_{v n}^{\text {(Cor })=} \frac{4}{\hbar^{2}}\left(\frac{\hbar}{2 I_{x}}\right)^{2}\left(\frac{V_{i} \hbar^{2}}{4}\right) \times \\
& \sum_{i \alpha}\left(\left|\zeta_{i \alpha}^{(x)}\right|^{2}+\left|\zeta_{i \alpha}^{(y)}\right|^{2}\right)\left(2+\frac{\omega_{i}}{\omega_{\alpha}}+\frac{\omega_{\alpha}}{\omega_{i}}\right) \times \\
& \operatorname{Re} \int_{0}^{\infty} \mathrm{d} t \exp \left[t\left(-i \omega_{i}+i \Delta \beta+i \omega_{\alpha}+\Gamma_{\alpha}\right)\right] \times \\
& G_{n_{\alpha}=1}(t) G_{n_{\beta}=2}(t) \prod_{\gamma}^{\gamma \neq \alpha, \beta} G_{n_{\gamma}=0}(t)
\end{aligned}
$$

The rotational dependence of SRVL electronic relaxation rates and radiative rates on this $J$ value range is small or negligible for $\mathrm{C}_{6} \mathrm{H}_{6} .{ }^{13}$ Thus we may expect that a plot of the inverse intensity 
of the $K=0$ lines after correcting the rotational Boltzmann factor including the $(2 J+1)$-fold $m$ degeneracy vs. $J(J+1)$ will be linear. These plots for odd $J$ and even $J$ are shown in Figure 2, $a$ and $b$, respectively. From this figure we can see that reasonable good linear relations have been obtained. It should be noted that as to be expected from eq 4.1, the slopes of these two straight lines are indeed equal.

Now if one vibrational quantum of the $\nu_{1}$ mode is added to $14^{1} 1^{2}$, then the density of states will increase at least two orders of magnitude, and both $W_{v n J K}^{(\mathrm{BO})}$ and $W_{v n J K}^{(\mathrm{CO})}$ will increase rapidly but usually at a rate slower than that of the increase in the density of states.

Whether one of them will become dominating or not depends on the rate of increase of $W_{v n J N}^{(\mathrm{BO})}$ and $S_{v n J K}^{(\mathrm{Cor})}$ with respect to the increase of vibrational excitation energy. The work in progress is to obtain the spectral shifts of the spectrum shown in Figure $1 \mathrm{~b}$ and to carry out the theoretical analysis of these shifts in order to determine the magnitude of the coupling matrix elements involved in IVR.

\section{Appendix}

In the Appendix, we are concerned with the calculation of $G_{r_{2}}(t)$ for the case of a vibrational frequency shift. Notice that applying the Mehler formula

$$
\begin{aligned}
& \sum_{n=0}^{\infty} \frac{\exp [-(n+1 / 2) t]}{\pi^{1 / 2} 2^{n} n !} H_{n}(x) H_{n}(x) \exp \left[-1 / 2\left(x^{2}+x^{2}\right)\right]= \\
& (2 \pi \sinh t)^{-1 / 2} \exp \left[-1 / 4(x+x)^{2} \tanh (t / 2)-1 / 4(x-x)^{2}\right. \\
& \operatorname{coth}(t / 2)](\text { A. } 1)
\end{aligned}
$$

to eq 3.6 , we obtain

(13) W. E. Henke, H. L. Selzle, T. R. Hays, E. W. Schlag, and S. H. Lin, J. Chem. Phys., 76, 1335 (1982).

(1982).

$$
\begin{array}{r}
G_{n_{\gamma}}(t)= \\
E_{n_{\gamma}}(t) \frac{\beta_{\gamma}{ }^{\prime}}{\left(2 \pi \sinh \lambda_{\gamma}\right)^{1 / 2}} \int_{-\infty}^{+\infty} \int \mathrm{d} Q_{\gamma} \mathrm{d} \bar{Q}_{\gamma} X_{v n_{\gamma}}\left(Q_{\gamma}\right) \times \\
X_{v^{\prime} n_{\gamma}}\left(\bar{Q}_{\gamma}\right) \exp \left[-\frac{\beta_{\gamma}{ }^{\prime 2}}{4}\left\{\left(Q_{\gamma}+\bar{Q}_{\gamma}\right)^{2} \tanh \frac{\lambda_{\gamma}{ }^{\prime}}{2}+\left(Q_{\gamma}-\right.\right.\right. \\
\left.\left.\left.\bar{Q}_{\gamma}\right)^{2} \operatorname{coth} \frac{\lambda_{\gamma}{ }^{\prime}}{2}\right\}\right]
\end{array}
$$

where

$$
\begin{gathered}
\beta_{\gamma}{ }^{2}=\omega_{\gamma}{ }^{\prime} / \hbar \\
\lambda_{\gamma}{ }^{\prime}=t\left(\Gamma_{\gamma}-i \omega_{\gamma}{ }^{\prime}\right) \\
E_{n_{\gamma}}(t)=\exp \left[-i t \omega_{\gamma}\left(n_{\gamma}+1 / 2\right)-\left(n_{\gamma}-1 / 2\right) \Gamma_{\gamma} t\right]
\end{gathered}
$$

Using the contour integral representation for the Hermite polynomial

$$
H_{n}(x)=(-1)^{n} \frac{n !}{2 \pi i} \int_{c} \frac{\mathrm{d} z}{z^{n+1}} \exp \left(-z^{2}-2 x z\right)
$$

we can carry out the integrations over $Q$ and $\bar{Q}_{\gamma}$ in eq A.2

$$
\begin{aligned}
G_{n_{\gamma}}(t)= & G_{n_{\gamma}=0}(t) \frac{E_{n_{\gamma}}(t)}{E_{n_{\gamma}=0}(t)} \frac{n_{\gamma} !}{2^{n_{\gamma}}}\left(\frac{1}{2 \pi i}\right)^{2} \times \\
& \int_{c} \int_{c} \frac{\mathrm{d} z_{1} \mathrm{~d} z_{2}}{z_{1}{ }^{n_{\gamma}+1} z_{2}{ }^{n_{\gamma}+1}} \exp \left(-a_{\gamma} z_{1}^{2}-a_{\gamma} z_{2}^{2}+b_{\gamma} z_{1} z_{2}\right)
\end{aligned}
$$

$a_{\gamma}$ and $b_{\gamma}$ are given in eq 3.12 and 3.13, respectively. Performing the contour integrations in eq A.7 we obtain $G_{n_{\gamma}}(t)$ given by eq 3.7 .

Acknowledgment. The authors thank Professor R. A. Marcus for helpful discussions. This work was supported in part by the NSF US-Germany program.

Registry No. Benzene, 71-43-2.

\title{
Angular Distribution of the Desorption of Carbon Dioxide Produced on Well-Polished Polycrystalline Iridium Surfaces
}

\author{
Tatsuo Matsushima \\ Research Institute for Catalysis, Hokkaido University, Sapporo 060, Japan (Received: May 23, 1983; \\ In Final Form: August 22, 1983)
}

\begin{abstract}
The angular distribution of the desorption of $\mathrm{CO}_{2}$ produced on well-polished polycrystalline iridium surfaces was determined by means of angle-resolved thermal desorption. $\mathrm{CO}_{2}$ generated by heating the sample covered by oxygen and $\mathrm{CO}$ peaked around $400 \mathrm{~K}$. The angular distribution of the desorption of the product $\mathrm{CO}_{2}$ varies as $(\cos \theta)^{3}$, where $\theta$ is the desorption angle. This indicates that $\mathrm{CO}_{2}$ leaves the surface immediately after the formation.
\end{abstract}

\section{Introduction}

The angular distribution of the desorption flux of product molecules gives microscopic insight into the dynamics of surface reactions. ${ }^{1,2}$ This determination is the first step in analysis of translational energies of desorbing molecules. The desorption flux of product has been angle resolved in the oxidation of $\mathrm{CO}$ and $\mathrm{H}_{2}$ on platinum metals. The desorption of the product $\mathrm{H}_{2} \mathrm{O}$ on $\mathrm{Pd}(111)$ and $\mathrm{Pt}(111)$ surfaces obeys a simple cosine law. ${ }^{3-5}$ On

(1) W. van Willigen, Phys. Lett. A, 28, 80 (1968).

(2) G. Comsa, Proc. Int. Vac. Congr., 7th, 1317 (1977).

(3) J. N. Smith, Jr., and R. L. Palmer, J. Chem. Phys., 56, 13 (1972). the other hand, the product $\mathrm{CO}_{2}$ desorption shows a very sharp angular distribution along the surface normal on $\operatorname{Pt}(111)^{6,7}$ and polycrystalline surfaces. ${ }^{8}$ It, however, obeys the cosine law on Pd(111), ${ }^{9}$ although no essential difference has been observed in

(4) T. Engel and H. Kuipers, Surf. Sci., 90, 181 (1979).

(5) C. T. Ceyer, W. L. Guthrie, T. H. Lin, and G. A. Somorjai, J. Chem. Phys., 78, 6982 (1983).

(6) R. L. Palmer and J. N. Smith, Jr., J. Chem. Phys., 60, 1453 (1974). (7) C. T. Campbell, G. Ertl, H. Kuipers, and J. Segner, J. Chem. Phys., 73, $5862(1980)$.

(8) C. A. Becker, J. P. Cowin, L. Wharton, and D. J. Auerbach, J. Chem. Phys, 67, 3394 (1977). 